\title{
Spectroscopic Studies of Fluorescence Effects in Bioactive 4-(5-Heptyl-1,3,4-Thiadiazol-2-yl)Benzene-1,3-Diol and 4-(5-Methyl-1,3,4-Thiadiazol-2-yl)Benzene-1,3-Diol Molecules Induced by $\mathbf{p H}$ Changes in Aqueous Solutions
}

\author{
Arkadiusz Matwijczuk $^{1}$ - Dariusz Kluczyk ${ }^{2}$ • Andrzej Górecki ${ }^{3}$ • \\ Andrzej Niewiadomy ${ }^{4,5} \cdot$ Mariusz Gagoś $^{2}$
}

Received: 7 November 2016 / Accepted: 19 February 2017 / Published online: 1 March 2017

(C) The Author(s) 2017. This article is published with open access at Springerlink.com

\begin{abstract}
This paper presents the results of stationary fluorescence spectroscopy and time-resolved spectroscopy analyses of two 1,3,4-thiadiazole analogues, i.e. 4-(5-methyl-1,3,4thiadiazol-2-yl)benzene-1,3-diol (C1) and 4-(5-heptyl-1,3,4thiadiazol-2-yl)benzene-1,3-diol (C7) in an aqueous medium containing different concentrations of hydrogen ions. An interesting dual florescence effect was observed when both compounds were dissolved in aqueous solutions at $\mathrm{pH}$ below 7 for $\mathrm{C} 1$ and 7.5 for $\mathrm{C} 7$. In turn, for $\mathrm{C} 1$ and $\mathrm{C} 7$ dissolved in water at $\mathrm{pH}$ higher than the physiological value (mentioned above), single fluorescence was only noted. Based on previous results of investigations of the selected 1,3,4-thiadiazole compounds, it was noted that the presented effects were associated with both conformational changes in the analysed
\end{abstract}

Electronic supplementary material The online version of this article (doi:10.1007/s10895-017-2053-y) contains supplementary material, which is available to authorized users.

Arkadiusz Matwijczuk

arkadiusz.matwijczuk@up.lublin.pl; arekmatwijczuk@gmail.com

$\triangle$ Mariusz Gagoś

mariusz.gagos@poczta.umcs.lublin.pl

1 Department of Biophysics, University of Life Sciences in Lublin, Akademicka 13, 20-950 Lublin, Poland

2 Department of Cell Biology, Institute of Biology, Maria Curie-Skłodowska University, 20-033 Lublin, Poland

3 Department of Physical Biochemistry, Faculty of Biochemistry, Biophysics and Biotechnology of the Jagiellonian University, Gronostajowa 7, 30-387 Krakow, Poland

4 Institute of Industrial Organic Chemistry, Annopol 6, 03-236 Warsaw, Poland

5 Department of Chemistry, University of Life Sciences in Lublin, 20-950 Lublin, Poland molecules and charge transfer (CT) effects, which were influenced by the aggregation factor. However, in the case of $\mathrm{C} 1$ and $\mathrm{C} 7$, the dual fluorescence effects were visible in a higher energetic region (different than that observed in the 1,3,4thiadiazoles studied previously). Measurements of the fluorescence lifetimes in a medium characterised by different concentrations of hydrogen ions revealed clear lengthening of the excited-state lifetime in a $\mathrm{pH}$ range at which dual fluorescence effects can be observed. An important finding of the investigations presented in this article is the fact that the spectroscopic effects observed not only are interesting from the cognitive point of view but also can help in development of an appropriate theoretical model of molecular interactions responsible for the dual fluorescence effects in the analysed 1,3,4thiadiazoles. Furthermore, the study will clarify a broad range of biological and pharmaceutical applications of these compounds, which are more frequently used in clinical therapies.

Keywords Dual fluorescence effects $\cdot$ Molecular spectroscopy $\cdot$ Molecular aggregation $\cdot$ Substituent effects . 1,3,4-thiadiazole

\section{Introduction}

As reported by the World Health Organisation, one of the major challenges of modern medicine is the fight against cancer and neurodegenerative diseases. According to literature data, these diseases are currently the leading causes of patients' mortality worldwide [1,2]. Great hopes in the fight against cancer and neurodegenerative diseases are placed on synthetic compounds from the 1,3,4-thiadiazole group, whose different derivatives are already known and clinically applied. The 1,3,4-thiadiazoles with a substituted resorcyl fragment 
analysed in this study are highly promising compounds in anticancer and neurodegenerative diseases. As indicated in most research papers, 1,3,4-thiadiazoles are the most attractive neuroprotective-activity molecule system of all the other thiadiazole systems [3-5]. Many compounds of this group have been used in medicine as e.g. oxidation inhibitors, colourants, and metal complexing compounds [6]. Additionally, the literature shows that the thiadiazole family comprises compounds with antitumour [7-10], antifungal [11], antibacterial [11], anti-inflammatory [12], anticonvulsant [13], antiviral [14], antituberculosis [15], antihypertensive [16], and antidepressant [17] activity.

Two highly promising 1,3,4-thiadiazole analogues with a confirmed neuroprotective activity, i.e. 4-(5-methyl-1,3,4thiadiazol-2-yl)benzene-1,3-diol (C1) (C1, Scheme 1A-C) and 4-(5-heptyl-1,3,4-thiadiazol-2-yl)benzene-1,3-diol (C7) (C7, Scheme 1D-F), were selected in this study for the investigations of the mechanism of molecular interactions. Noteworthy, these 1,3,4-thiadiazole compounds exhibit not only remarkable and confirmed pharmacological properties but also very interesting spectroscopic traits, which contribute to their biological activity. The spectroscopic effects exhibited by the 1,3,4-thiadiazole analogues include e.g. the effects of keto/enol tautomerism induced by changes in medium polarizability [18-21], effects associated with crystal polymorphism [22] and solvatomorphism [23], and interesting interactions in model lipid systems [24, 25]. Additionally, the analysed compound group exhibits an interesting dual fluorescence effect or an effect of several fluorescence spectra, depending on the concentration [26], $\mathrm{pH}$, or changes in the medium temperature, which will be presented in this paper [27]. Moreover, these compounds are good ligands, which can form complexes with d-block metal ions [28], and can therefore be used in novel medical applications. The combination of the spectroscopic and crystallographic effects reported in the papers cited above has great importance for elucidation of the broad spectrum of the pharmacological activity of these compounds.

The aim of the present spectroscopic study was to investigate $\mathrm{C} 1$ and $\mathrm{C} 7$ in a medium with different concentrations of hydrogen ions and to describe the dual fluorescence effect observed in these molecules. In previous studies, the dual
Scheme 1 Chemical structure of the $\mathrm{C} 1$ molecule $(\mathbf{a}$ - enol form, $\mathbf{b}$ - form ionised with the $-\mathrm{O}^{-}$ group, $\mathbf{c}-$ form ionised with the$\mathrm{N}^{+}-\mathrm{H}$ group) and $\mathrm{C} 7$ molecule $(\mathbf{d}$ - enol form, $\mathbf{e}-$ form ionised with the $-\mathrm{O}^{-}$group, $\mathbf{f}-$ form ionised with the $-\mathrm{N}^{+}-\mathrm{H}$ group)<smiles>Cc1nnc(-c2ccc(O)cc2O)s1</smiles><smiles>Cc1nnc(-c2ccc(O)cc2[O-])s1</smiles> 
fluorescence effect was observed for one representative of the analogue group (FABT) [27]. However, in this study, the effect was observed in a different (substantially higher) energy range, which completely changed the photophysical properties of the analysed molecules. Using spectroscopic approaches, such as the electron absorption spectroscopic technique, fluorescence methods combined with the RLS technique, and mainly measurements of fluorescence lifetimes, we have shown the complexity of physical processes that exert an impact on the observed effects. The dual fluorescence effects can be induced in different molecules by changes in medium polarity, $\mathrm{pH}$ of the solution, temperature, or the concentration of the sample [29-33]. According to theories attempting at elucidation of the observed fluorescence effects, they can be related to appearance of intramolecular CT states [34] and the so-called TICT states (Twisted Intramolecular Charge Transfer) $[35,36]$. Another explanation of the analysed phenomena is the process of Excited-State Intramolecular Proton Transfer ESIPT [37-42]. Other theories postulate formation of compound concentration-induced excimer systems [43, 44], various types of acid-base reactions in the investigated system, or very frequent formation of excited-state tautomeric systems [45, 46]. The anti-Kasha mechanism of the dual fluorescence effect postulated in 2015 in the Journal of Physical Chemistry B by Brancato et al. should also be mentioned [47]. However, as suggested by the research results presented in this paper, none of these mechanisms seems to be successful in elucidation of the observed effects and molecular mechanisms involved in the spectroscopic changes.

The studies performed with the use of stationary fluorescence spectroscopy and time-resolved spectroscopy in an aqueous medium with different $\mathrm{pH}$ values revealed the dual fluorescence effect in both analogues.

Formulation of a concise theory for the dual fluorescence effects observed in the 1,3,4-thiadiazole analogues is crucial for understanding their biological activity. The effect is attractive for theoretic reasons, as it can be used for examination of the excitation state in various molecular transformations and for designing new molecular probes of (dual) fluorescence.

\section{Material and Methods}

\section{Materials}

4-(5-methyl-1,3,4-thiadiazol-2-yl)benzene-1,3-diol (C1) (see Scheme 1D) and 4-(5-heptyl-1,3,4-thiadiazol-2-yl)benzene1,3-diol (C7) were synthesized in the Department of Chemistry of the University of Life Sciences in Lublin, details of the procedure are described elsewhere [3]. The purification procedure of the $\mathrm{C} 1$ and $\mathrm{C} 7$ compound is described in detail in the references [3]. The concentration of the compounds was $\mathrm{c}=1.25 \times 10^{-6} \mathrm{M}$ for $\mathrm{C} 1$ and $\mathrm{c}=1.19 \times 10^{-6} \mathrm{M}$ for $\mathrm{C} 7$.

\section{Methods}

\section{pH Measurement and Preparation}

All solutions were measured with an Elmetron CP-502 pHmeter at room temperature. In the case of the aqueous $\mathrm{C} 1$ and C7 solutions, $0.1 \mathrm{M} \mathrm{NaOH}$ was first added to water to obtain $\mathrm{pH}$ 12. Afterwards, powder $\mathrm{C} 1$ and $\mathrm{C} 7$ was dissolved therein. Next, $0.1 \mathrm{M} \mathrm{HCl}$ acid was slowly added to obtain a certain $\mathrm{pH}$ value in the water $\mathrm{C} 1$ and $\mathrm{C} 7$ solution. The $\mathrm{pH}$ was continually controlled. Respective titration curves for both 1,3,4thiadiazole analogues are shown in Fig. S1 in the Supplementary Materials. The insets in Fig. S1 present ionised functional groups and $\mathrm{pK}$ points for both compounds (see below).

\section{Electronic Absorption Spectroscopy}

Electronic absorption spectra of $\mathrm{C} 1$ and $\mathrm{C} 7$ were recorded on a double-beam UV-Vis spectrophotometer Cary 300 Bio (Varian) equipped with a thermostatted cuvette holder with a $6 \times 6$ multicell Peltier block. Temperature was controlled with a thermocouple probe (Cary Series II from Varian) placed directly in the sample. All experiments were carried out at $23^{\circ} \mathrm{C}$. The spectral slit width was $1.5 \mathrm{~nm}$ in the measurements of the electron absorption spectra.

\section{Electronic Fluorescence Spectroscopy with the RLS Technique}

Fluorescence excitation and emission as well as synchronous spectra were recorded with a Cary Eclipse spectrofluorometer (Varian) at $23{ }^{\circ} \mathrm{C}$. Fluorescence spectra were recorded with $0.5 \mathrm{~nm}$ resolution and corrected for the lamp and photomultiplier spectral characteristics. Resonance light scattering (RLS) measurements were performed as in Pasternack and Collings [48, 49]. The excitation and emission monochromators of the spectrofluorimeter were scanned synchronously $(0.0 \mathrm{~nm}$ interval between excitation and emission wavelengths), the slits were set to obtain spectral resolution of $1.5 \mathrm{~nm}$. The spectral analysis was performed with the use of Grams/AI 8.0 software (Thermo Electron Corporation).

\section{Time-Correlated Single Photon Counting (TCSPC)}

Time-correlated single photon counting (TCSPC) measurements were performed on a FluoroCube fluorimeter (Horiba, France). The samples were excited with a pulsed NanoLED diode at $372 \mathrm{~nm}$ (pulse duration of $150 \mathrm{ps)} \mathrm{operated} \mathrm{with}$ $1 \mathrm{MHz}$ repetition. To avoid pulse pile-up, the power of the 
pulses was adjusted to an appropriate level using a neutral gradient filter. Fluorescence emission was recorded using a picosecond detector TBX-04 (IBH, JobinYvon, UK). The DataStation and the DAS6 software (JobinYvon (IBH, UK)) were used for data acquisition and signal analysis. All fluorescence decays were measured in a $10 \times 10 \mathrm{~mm}$ quartz cuvette, using an emitter cut-off filter with transmittance for wavelengths longer than $408 \mathrm{~nm}$. The excitation profiles required for the deconvolution analysis were measured without the emitter filters on a light scattering cuvette. All measurements were performed in water at $20{ }^{\circ} \mathrm{C}$ and various $\mathrm{pH}$. Fluorescence decay was analysed with a multiexponential model given by the equation:

$I_{t}=\sum_{i} \alpha_{i} \exp \left(-\frac{t}{\tau}\right)$

where $\alpha_{\mathrm{i}}$ and $\tau_{\mathrm{i}}$ are the pre-exponential factors and the decay time of component $i$, respectively.

Best-fit parameters were obtained by minimization of the reduced $\chi[2]$ value as well as residual distribution of experimental data. The average lifetime of fluorescence decay was calculated according to the following equation:

$\langle\tau\rangle=\frac{\Sigma_{i} \alpha_{i} \tau_{i}^{2}}{\Sigma_{i} \alpha_{i} \tau_{i}}$

\section{Results and Discussion}

By plotting the $\mathrm{pH}$ titration curves for $\mathrm{C} 1$ and $\mathrm{C}$, characteristic $\mathrm{pK}$ points for the ionisation-associated groups were determined in the studied 1,3,4-thiadiazoles (Fig. S1 in the Supplementary Materials). For the $-\mathrm{O}^{-}$group in the ortho position in the resorcyl ring, $\mathrm{pK}_{\mathrm{C} 7}=8.8$ and $\mathrm{pK}_{\mathrm{C} 1}=8.1$ (Fig. S1 in the Supplementary Materials - figure insets) and for the $-\mathrm{NH}^{+}$group, $\mathrm{pK}_{\mathrm{C} 7}=4.9$ and $\mathrm{pK}_{\mathrm{C} 1}=4.1$ (Fig. $\mathrm{S} 1$ in the Supplementary Materials - figure insets).

Panels $\mathrm{A}$ and $\mathrm{C}$ in Fig. 1 present results obtained with electron absorption spectroscopy for C1 (Panel A) and C7

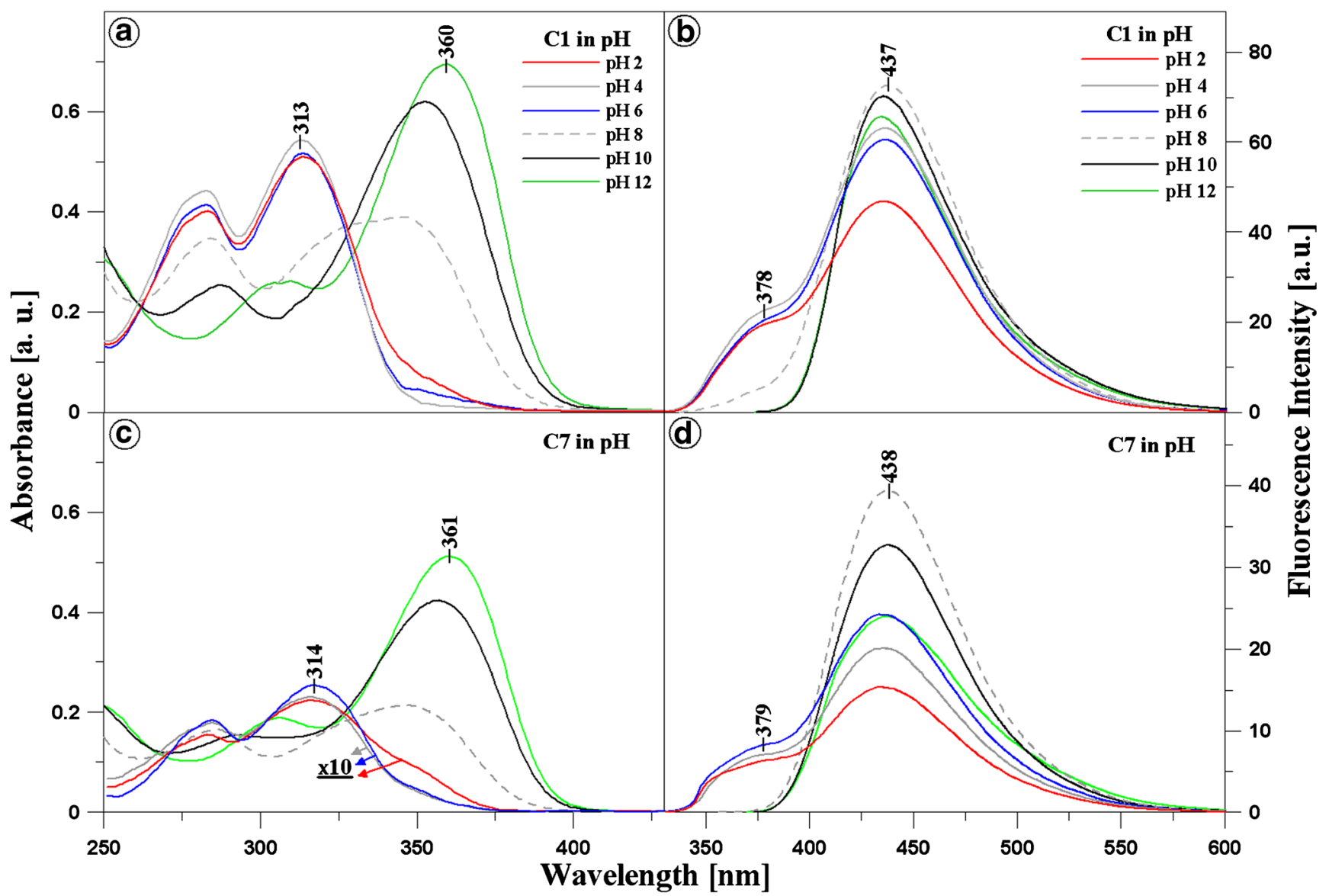

Fig. 1 Panels $\mathrm{A}$ and $\mathrm{C}$ present electron absorption spectra for $\mathrm{C} 1$ (Panel $A$ ) and $\mathrm{C} 7($ Panel $C)$ generated in the aqueous solution at $\mathrm{pH} 12,10,8,6$, 4, and 2. For C7 in Panel C, the absorption spectra at $\mathrm{pH} 2$, 4, and 6 were multiplied by 10 for better presentation and comparison of the analysed

effects. Panels B and D show fluorescence emission spectra corresponding to the spectra from Panels A and C for $\mathrm{C} 1$ (Panel B) and $\mathrm{C} 7$ (Panel D). The excitation wavelength corresponded to the maximum of the respective absorption band 
(Panel C) over the entire range of hydrogen ion concentrations (from $\mathrm{pH} 1$ to $\mathrm{pH}$ 12). There results show distinct changes in the shape of the spectra, in particular in the region that is relevant to the physiological values. Spectra for $\mathrm{pH} 2,4,6$, 8,10 , and 12 for both analysed compounds are presented for better clarity. The spectra in the $\mathrm{pH}$ range from 1 to 6 were multiplied by 10 (specifically: for $\mathrm{C} 7$ at $\mathrm{pH} \mathrm{2,} \mathrm{4,} \mathrm{and} \mathrm{6)} \mathrm{for}$ clearer presentation of the analysed effects for $\mathrm{C} 7$ (Panel $\mathrm{C}$ in Fig. 1). As shown in both panels in Fig. 1, the dissociation of the $-\mathrm{OH}$ group from the resorcyl ring in the ortho position (Scheme 1B and E) results in a clear hypsochromic shift by $301 \mathrm{~cm}^{-1}$ in the case of $\mathrm{C} 7$ and by $303 \mathrm{~cm}^{-1}$ for $\mathrm{C} 1$ in the compound spectra at $\mathrm{pH} 12$. In addition, there is a bathochromic shift by $3845 \mathrm{~cm}^{-1}$ for $\mathrm{C} 7$ and $3864 \mathrm{~cm}^{-1}$ for $\mathrm{C} 1$ for spectra of both compounds at $\mathrm{pH} 1$, compared with their spectra at $\mathrm{pH}$ 7. These shifts are used for calculation of the distances between the molecules in the dimer using the exciton splitting theory. In both 1,3,4-thiadiazole analogues, the ionisation process is accompanied by compound aggregation [27]. At $\mathrm{pH}$ ca. 7-8, distinct broadening of the absorption spectra is evident for $\mathrm{C} 1$ and $\mathrm{C} 7$ (dashed grey line in Panels $\mathrm{A}$ and C, Fig. 1), which indicates a probability of the presence of other than monomeric spectral forms of the analysed structures [27]. In the case of the $\mathrm{C} 7$ spectrum at $\mathrm{pH} 2$, the absorbance is the lowest, which implies substantial predominance of aggregated forms in this compound (see Panel B in Fig. 2). In turn, in the case of $\mathrm{C} 1$, the lowest absorbance intensity is observed in the same range, but it is substantially higher than that for $\mathrm{C} 7$ at the corresponding $\mathrm{pH}$. This indicates an impact of the structure of the analysed compounds, in particular the structure of their alkyl substituents, on the mode of formation of aggregated forms of these molecules. The structure of substituent groups can have a significant influence (through processes related to stronger aggregation) on the solubility of the analysed molecules in different organic solvents. For $\mathrm{C} 1$ at $\mathrm{pH} 4$, the compound absorbance unexpectedly increases slightly, suggesting an increase in the number of the monomeric forms of this analogue. No such phenomenon is observed in the case of $\mathrm{C} 7$ in the specified concentration range, which evidences stronger interactions between $\mathrm{C} 7$ molecules and formation of more durable aggregates. In $\mathrm{C} 7$, a remarkable decrease in the absorbance level is visible, which implies very strong aggregation at a (probably) constant level along the decrease in the $\mathrm{pH}$ value (at low $\mathrm{pH}$ ).

Based on the exciton splitting theory and the spectral shifts presented in Fig. 1 and above (and in Fig. S2 in the Supplementary Materials), it was possible to calculate the distance between adjacent chromophores of molecules $\mathrm{C} 1$ and C7 in the dimeric structure [49]. Fig. S2 (in the Supplementary Materials) shows electron absorption spectra for C7 (Panel A) and $\mathrm{C} 1$ (Panel B) normalised at the maximum. Underpinning of the band can be observed for both $\mathrm{C} 1$ and $\mathrm{C} 7$ at $\mathrm{pH}$ ca. 1, compared with $\mathrm{pH}$ 7. For C7 (Panel A), the band with a

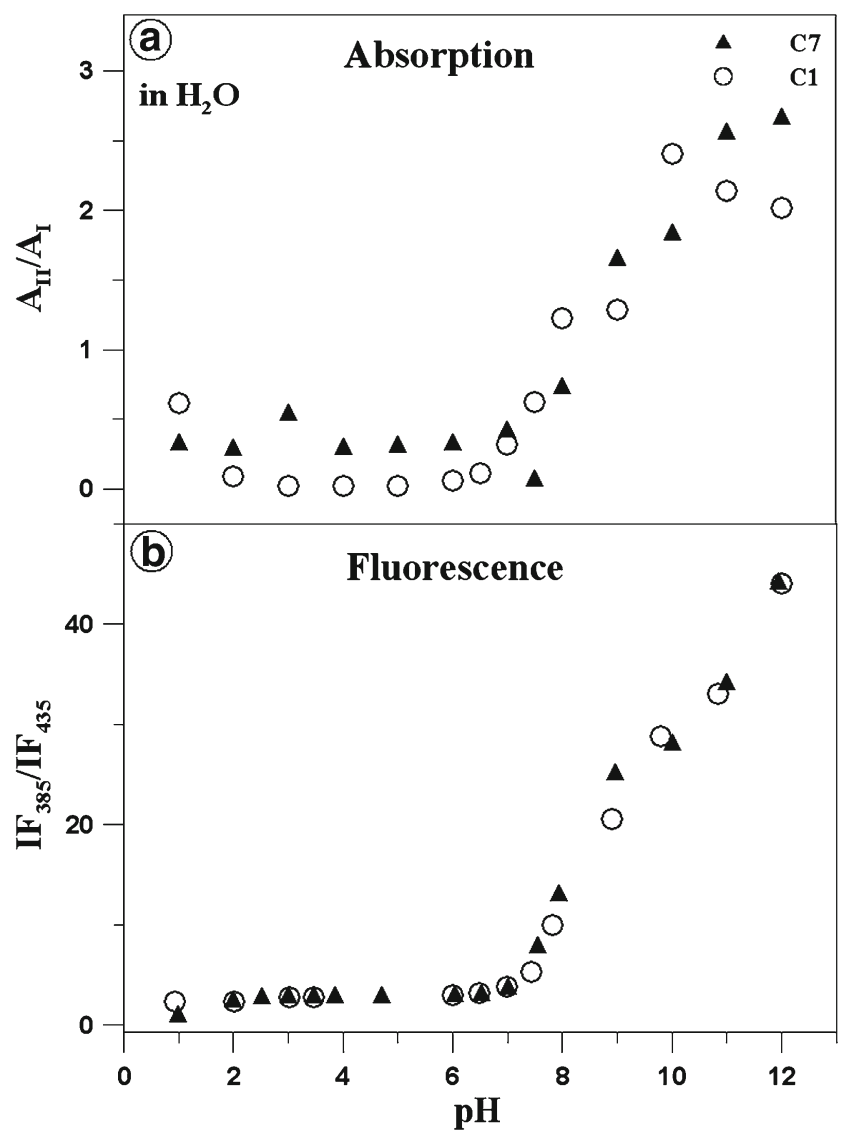

Fig. 2 Panel A presents the ratio of the maximum electron absorption at ca. $360 / 313 \mathrm{~nm}$ for $\mathrm{C} 1$ (white circles) and at $361 / 314 \mathrm{~nm}$ for C7 (black triangles), i.e. the ratio between the predominant monomeric form (ionised with the $-\mathrm{O}^{-}$group) and the predominant associated form for a given compound (ionised with the $-\mathrm{N}-\mathrm{H}^{+}$group) depending on the $\mathrm{pH}$ of the aqueous solution. Panel $\mathrm{B}$ shows the ratio of the fluorescence emission intensity for $\mathrm{C} 1$ (white circles) and $\mathrm{C} 7$ (black triangles) depending on changes in the $\mathrm{pH}$ of the aqueous solution. The points were read from the absorption and fluorescence emission spectra presented in Fig. 1. The measurements of the absorption and electron fluorescence spectra, from which the respective absorption and fluorescence maxima were read

maximum at ca. $319 \mathrm{~nm}\left(31,348 \mathrm{~cm}^{-1}\right)$ at $\mathrm{pH} 7$ is slightly shifted towards $317 \mathrm{~nm}\left(31,546 \mathrm{~cm}^{-1}\right)$ and a band with a maximum at ca. $351 \mathrm{~nm}\left(28,490 \mathrm{~cm}^{-1}\right)$ appears on the longwave side. Similarly, in the case of $\mathrm{C} 1$, the main bands with a maximum at ca. $316 \mathrm{~nm}\left(31,646 \mathrm{~cm}^{-1}\right)$ is shifted at low $\mathrm{pH}$ values towards ca. $314 \mathrm{~nm}\left(31,847 \mathrm{~cm}^{-1}\right)$ and a band with a maximum at ca. $357 \mathrm{~nm}\left(28,011 \mathrm{~cm}^{-1}\right)$ can be seen on the longwave side.

Based in the exciton splitting theory, the distance between adjacent chromophores $\mathrm{R}_{\beta}$ can be calculated using the formula:

$R_{\beta}=1.71 \sqrt[3]{\frac{\mu^{2} \kappa}{\eta^{2} \beta}}$

where $\mu$ is the diploe moment of transition of the interacting molecules, $\eta$ - refractive index, $\beta$ - dipole-dipole interaction 
energy (in a classical approach). In the excitonic model, one can consider an aggregated structure formed through interaction of identical molecules, in which transition dipole moments of adjacent molecules are parallel, hence $\alpha=0$ (where, $\kappa=1-3 \cos [2] \theta$, where $\theta$ is the angle between the transition dipole moments). As proposed in the exciton splitting theory $[50,51], \kappa=1$ for the card pack molecule arrangement in the aggregate and $\mathrm{k}=-2$ for the head to tail aggregate. The transition dipole moment calculated via integration of the absorption spectrum is $\mu=4.16 \mathrm{D}$ (in $\mathrm{H}_{2} \mathrm{O}$ ) and $\mu=4.25 \mathrm{D}$ for $\mathrm{C} 7$ (the values of transition dipole moments in other solvents and water as well as the molar value of the extinction coefficients are presented in Table S1 in the Supplementary Materials). The calculated distance between adjacent chromophores is $4.29 \AA^{50}$ in the case of the $\mathrm{C} 1$ dimers in an aqueous solution and $3.87 \AA$ for $\mathrm{C} 7$. These results are consistent with crystallographic data presented in some other 1,3,4thiadiazole compounds [27]. The distance in crystals is lower than in solutions, which is the cause of the obviously denser packing of the analysed molecules in the crystalline structure and stronger intermolecular interactions. However, the most important fact is that the distance between adjacent molecules in $\mathrm{C} 7$ is substantially smaller than in $\mathrm{C} 1$.

Panel A in Fig. 2 shows the ratio of the absorbance maximum at $360 \mathrm{~nm}$ (predominant monomeric form, Scheme 1B, and $\mathrm{E}$ - ionised with the $-\mathrm{O}^{-}$group) and $313 \mathrm{~nm}$ (predominant associated form, Scheme $1 \mathrm{C}$, and $\mathrm{F}-$ ionised with the- $\mathrm{NH}^{+}$ group) for $\mathrm{C} 1$ (open circles) as well as the ratio of the absorbance maximum of $361 \mathrm{~nm}$ and $314 \mathrm{~nm}$ for $\mathrm{C} 7$ (black triangles), depending on the $\mathrm{pH}$ of the aqueous solution. The predominance of the negatively ionised form (predominance of the monomeric form) for both $\mathrm{C} 1$ and $\mathrm{C} 7$ compounds is most evident at the high $\mathrm{pH}$ values $(\mathrm{pH} 12$ for $\mathrm{C} 7$ and $\mathrm{pH} 10.5$ for $\mathrm{C} 1)$. In contrast, the predominance of the forms ionised with the $\mathrm{NH}^{+}$group can be observed at the low $\mathrm{pH}$ values (at $\mathrm{pH} 1$ for both $\mathrm{C} 7$ and $\mathrm{C} 1$ ). In an acidic environment, the presence of a positively charged $\mathrm{NH}^{+}$group in the thiadiazole ring should also lead to monomerisation. However, the presence of the $\mathrm{OH}$ groups in the resorcyl ring facilitates generation of hydrogen bonds not only with water but also with other molecules, resulting in their aggregation. In turn, the greatest changes in the presented ratio in both compounds can be observed for the physiological $\mathrm{pH}$ values, which can be clearly seen in the absorption spectra presented in Panels A and C of Fig. 1.

In the next step of the investigations, the compounds were analysed with the fluorescence spectroscopy methods. Noteworthy are the effects presented in Panels B and D of Fig. 1. The panels show fluorescence emission spectra for C1 (Panel B) and C7 (Panel D), corresponding to the absorption spectra presented in Fig. 1 (Panels A and C), together with the change in the $\mathrm{pH}$ value of the aqueous solution $(\mathrm{pH} 2,4,6$, 8 , and 10 are presented analogously as for the absorption spectra). The excitation wavelength for all the analysed samples corresponds to the maximum of respective absorption bands. A dual fluorescence effect with a maximum at ca. $380 \mathrm{~nm}$ and $440 \mathrm{~nm}$ in the $\mathrm{pH}$ range from 1 to 7.5 (Panels C and $\mathrm{D}$ ) is evident in the case of both compounds. Above $\mathrm{pH} 7.5$, single fluorescence with a maximum at $438 \mathrm{~nm}$ for $\mathrm{C} 7$ and $437 \mathrm{~nm}$ for $\mathrm{C} 1$ is observed. At $\mathrm{pH} 8$, only slight underpinning of the respective fluorescence emission spectrum is noted for $\mathrm{C} 1$. Additionally, it should be emphasised that the intensity of the fluorescence emission spectra for $\mathrm{C} 7$ is substantially lower than that for $\mathrm{C} 1$ in the range where the aforementioned effect can be observed (the same concentration of both analogues). This clearly indicates a considerably greater degree of aggregation in $\mathrm{C} 7$ than that in $\mathrm{C} 1$ and more intensive fluorescence emission decay. This was also observed in the calculations based on the exciton splitting theory (see above), where the distances between adjacent chromophores were markedly smaller in $\mathrm{C} 7$ than in $\mathrm{C}$.

Panel B in Fig. 2 shows the ratio of short- and longwave fluorescence emission intensity for both compounds (emission with a maximum of ca. $380 \mathrm{~nm}$ vs. emission with a maximum of ca. $440 \mathrm{~nm}$ ) depending on the $\mathrm{pH}$ changes in the aqueous solution. Evidently, in the $\mathrm{pH}$ range from 1 to 7.5 for $\mathrm{C} 7$ and $\mathrm{C} 1$, the points are arranged in one almost horizontal line, likewise for the spectra shown in Fig. 1 (Panels A and C). This implies that the process of nitrogen atom protonation is in equilibrium and does not change the intensity of the ratio in the analysed 1,3,4-thiadiazole analogues (Scheme 1C and F). At a $\mathrm{pH}$ value higher than 7.5 (for both compounds), the ratio increases significantly and the fluorescence at $378 / 9 \mathrm{~nm}$ disappears for both compounds.

Noteworthy, a longwave fluorescence band with a maximum at ca. $500 \mathrm{~nm}$ appeared in the case of another 1,3,4-thiadiazole described previously, i.e. FABT [27], in which the structural differences are related to the presence of the N-H group and a fluorobenzene ring. In turn, in the compounds analysed in this paper, the decrease in the $\mathrm{pH}$ value is accompanied by a shortwave fluorescence emission band with a maximum at ca. $380 \mathrm{~nm}$. Therefore, changes in the energy structure of the 1,3,4-thiadiazole molecules are observed. This effect can be associated with the presence of the amino N-H group in the FABT structure located at the 1,3,4-thiadiazole ring [27]. In the case of a 1,3,4-thiadiazole that structurally resembles FABT, although without the fluorobenzene fragment, and differs from $\mathrm{C} 1$ only in the $\mathrm{N}-\mathrm{H}$ group, a band with a maximum at ca. $380 \mathrm{~nm}$ is observed at high $\mathrm{pH}$ values (results submitted for publishing). In turn, acidification yielded a separate band at ca. $440 \mathrm{~nm}$ (as in the case of $\mathrm{C} 1$ and $\mathrm{C} 7$ at the high $\mathrm{pH}$ values). These considerations indicate an impact of the substituents in the 1,3,4-thiadiazole structure on the position of fluorescence bands despite the identical chromophore organisation (five conjugated double bonds) in the molecule. 


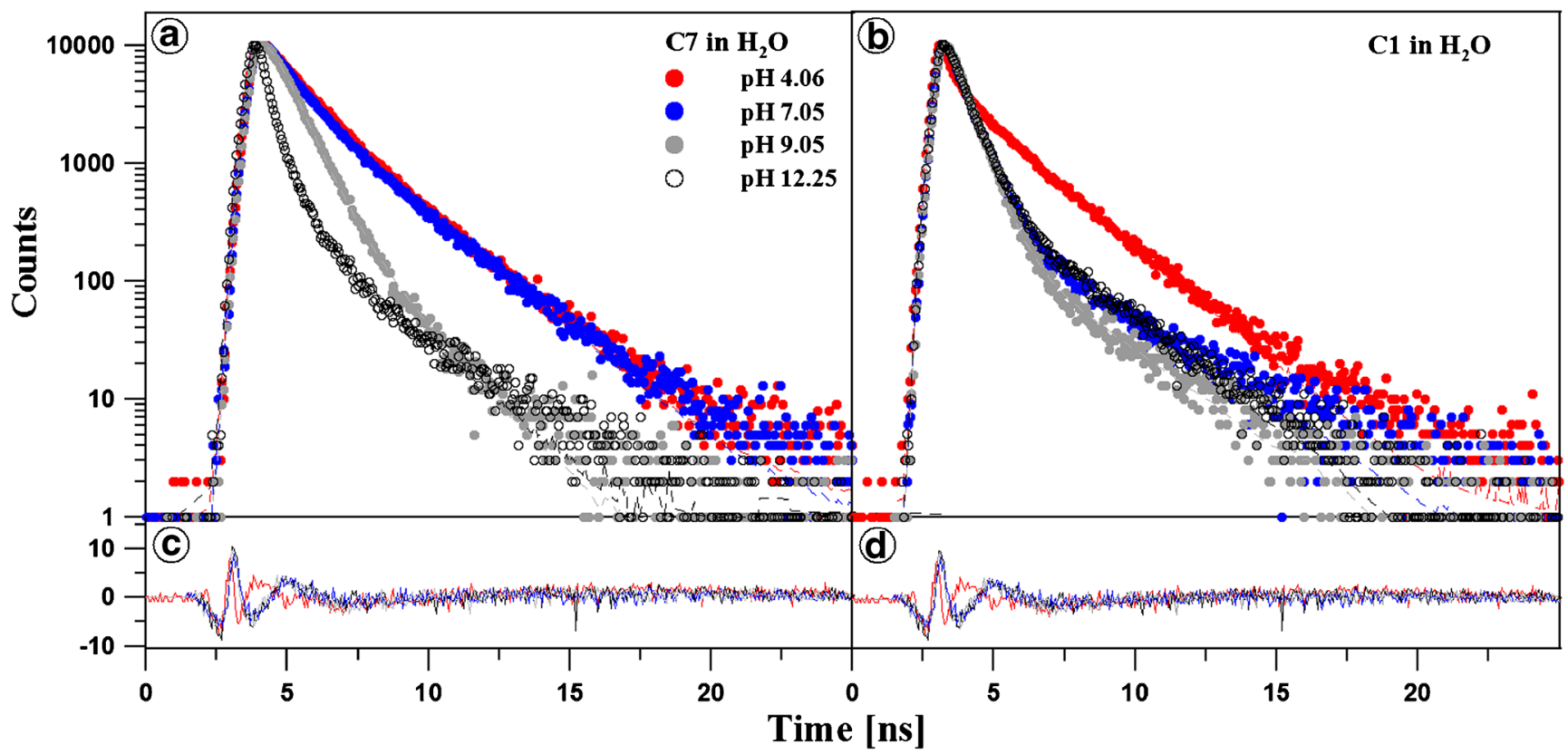

Fig. 3 The $\mathrm{pH}$ effect of the fluorescence decay of $\mathrm{C} 7$ and $\mathrm{C} 1$ in water. Dotted curves show the decay of fluorescence emission observed using the TCSPC technique at a given $\mathrm{pH}$ for $\mathrm{C} 7$ and $\mathrm{C} 1$ in Panels $\mathrm{A}$ and $\mathrm{B}$, respectively. Solid lines are best exponential fits. The excitation pulse

\section{Fluorescence Lifetime Study}

Figures 3 and 4 as well as Tables 1 and 2 present the results of measurements of fluorescence lifetimes for $\mathrm{C} 1$ and $\mathrm{C} 7$ in the aqueous solution over the entire $\mathrm{pH}$ range (shown for $\mathrm{pH} 4,7$, profile, set up at 372 , is shown by the dotted black curve. Residuals determined for the presented fits are shown below the decay curves in Panels C and D

9, and 12). Light with a wavelength of $372 \mathrm{~nm}$ was used for excitation. At pH 7, the selected excitation wavelength is typical for the longwave absorption edge of the monomeric form and resonant excitation of the aggregated form $(\sim 370 \mathrm{~nm})$. Fluorescence decay was monitored with the TCSPC method

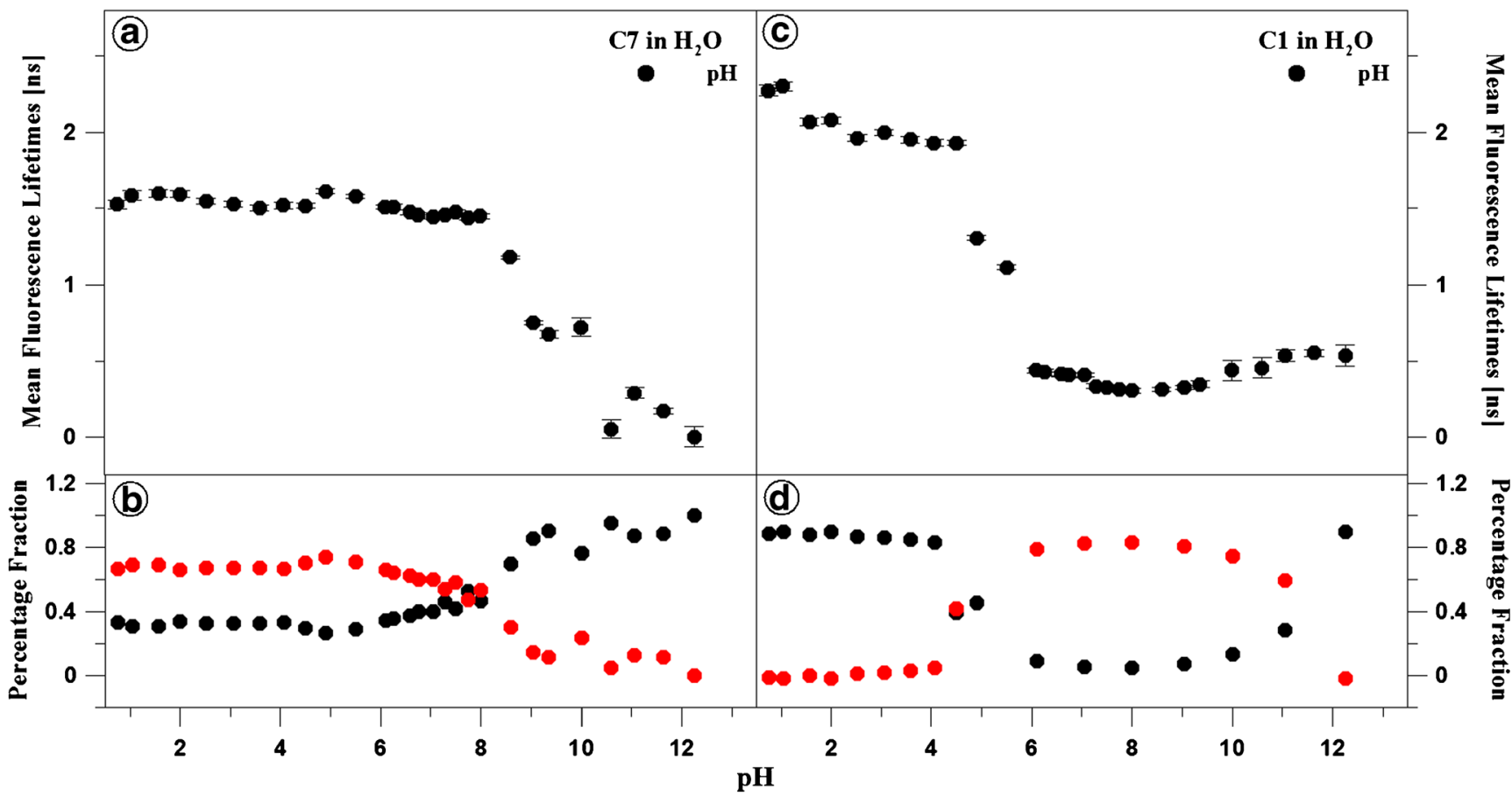

Fig. 4 The effect of $\mathrm{pH}$ on the fluorescence lifetime for $\mathrm{C} 7$ (Panels $\mathrm{A}$ and $B$ ) and $\mathrm{C} 1$ (Panels $C$ and $D$ ). The dependence of the mean fluorescence lifetimes on the best exponential fits at given $\mathrm{pH}$ are shown in Panels A and $\mathrm{C}$, while intensities of the fractional fluorescence lifetimes are presented in Panels B and D 
Table 1 Fluorescence lifetimes of $\mathrm{C} 7$ in $\mathrm{H}_{2} \mathrm{O}$ in relation to changes in $\mathrm{pH}$

Table 2 Fluorescence lifetimes of $\mathrm{C} 1$ in $\mathrm{H}_{2} \mathrm{O}$ in relation to changes in $\mathrm{pH}$

\begin{tabular}{|c|c|c|c|}
\hline $\mathrm{pH}$ & $\tau$ & \pm & $\Delta \tau$ \\
\hline 0.8 & 1.59 & \pm & 0.03 \\
\hline 1.0 & 1.59 & \pm & 0.03 \\
\hline 1.6 & 1.60 & \pm & 0.03 \\
\hline 2.0 & 1.59 & \pm & 0.02 \\
\hline 2.5 & 1.55 & \pm & 0.02 \\
\hline 3.1 & 1.53 & \pm & 0.02 \\
\hline 3.6 & 1.51 & \pm & 0.02 \\
\hline 4.1 & 1.52 & \pm & 0.02 \\
\hline 4.5 & 1.52 & \pm & 0.02 \\
\hline 4.9 & 1.61 & \pm & 0.02 \\
\hline 5.5 & 1.58 & \pm & 0.01 \\
\hline 6.1 & 1.51 & \pm & 0.01 \\
\hline 6.3 & 1.51 & \pm & 0.02 \\
\hline 6.6 & 1.47 & \pm & 0.01 \\
\hline 6.8 & 1.46 & \pm & 0.01 \\
\hline 7.1 & 1.44 & \pm & 0.01 \\
\hline 7.3 & 1.46 & \pm & 0.01 \\
\hline 7.5 & 1.48 & \pm & 0.01 \\
\hline 7.8 & 1.44 & \pm & 0.01 \\
\hline 8.0 & 1.45 & \pm & 0.01 \\
\hline 8.6 & 1.18 & \pm & 0.01 \\
\hline 9.1 & 0.75 & \pm & 0.01 \\
\hline 9.4 & 0.68 & \pm & 0.02 \\
\hline 10.0 & 0.72 & \pm & 0.06 \\
\hline 10.6 & 0.12 & \pm & 0.10 \\
\hline 11.1 & 0.29 & \pm & 0.03 \\
\hline 11.6 & 0.17 & \pm & 0.02 \\
\hline 12.3 & 0.11 & \pm & 0.07 \\
\hline
\end{tabular}

\begin{tabular}{llll}
\hline C1 & & & \\
\hline $\mathrm{pH}$ & $\tau$ & \pm & $\Delta \tau$ \\
\hline 1.0 & 2.45 & \pm & 0.06 \\
2.0 & 2.24 & \pm & 0.03 \\
3.0 & 2.17 & \pm & 0.01 \\
4.0 & 2.15 & \pm & 0.01 \\
5.0 & 1.29 & \pm & 0.01 \\
6.0 & 0.73 & \pm & 0.01 \\
7.0 & 0.63 & \pm & 0.01 \\
8.0 & 0.60 & \pm & 0.01 \\
9.0 & 0.62 & \pm & 0.01 \\
10.0 & 0.72 & \pm & 0.01 \\
11.0 & 0.81 & \pm & 0.04 \\
12.0 & 0.81 & \pm & 0.04 \\
\hline
\end{tabular}

at a temperature of $22{ }^{\circ} \mathrm{C}$ for light with a wavelength over $408 \mathrm{~nm}$. The results were analysed by deconvolution of the fluorescence decay using Eq. (1) each time for $i=1,2$, and 3. In a majority of the analysed cases, the double exponential model of fluorescence decay proved to be optimal. The mono-exponential decay model was insufficient, and addition of a third component did not improve the quality of the fit, which was verified by the value of the fit parameter and analysis of residue distribution (Fig. 3, Panels C and D). A single exponential was only observed for $\mathrm{C} 1$ at $\mathrm{pH}$ lower than 5 . In these cases, an additional component did not improve the fit. For C7, both components of the fluorescence lifetimes exhibited very little variability at $\mathrm{pH}$ below 8,5 . In this range, the lifetime of the longer component is between 2 and 2,5 ns and is rapidly reduced to ca. $1 \mathrm{~ns}$ over the threshold $\mathrm{pH}$ value. Its contribution is ca. $70 \%$ at the low $\mathrm{pH}$ values and declines to ca. $10 \%$ at the higher $\mathrm{pH}$ values. The lifetime of the second component is ca. $0.5 \mathrm{~ns}$ at the lower $\mathrm{pH}$ values and decreases to several tens of $\mathrm{ps}$ for the higher $\mathrm{pH}$ values. As a result of this variability, the average lifetime is ca. 1,6 ns for $\mathrm{pH}$ lower than 8 and is reduced to several tens of ps at the higher $\mathrm{pH}$ values. In the case of $\mathrm{C} 1$, the component with the longer lifetime has a similar value to that observed for $\mathrm{C} 7$ and is $2-$ 2,5 ns over the entire $\mathrm{pH}$ range. From $\mathrm{pH}$, a second component with a lifetime of $0,5 \mathrm{~ns}$ is observed and its value changes insignificantly over the analysed $\mathrm{pH}$ range. With the additional component, the average lifetime is markedly reduced at the $\mathrm{pH}$ increase up to ca. 6 and then remains relatively constant at ca. $0,5-1 \mathrm{~ns}$ up to the $\mathrm{pH}$ value of 11 .

Noteworthy, drastic changes in the length of the lifetimes are observed from a $\mathrm{pH}$ value of ca. 8 in the case of $\mathrm{C} 7$ (Fig. 4, Panel A) and from pH of ca. 5.5 in C1 (Fig. 4, Panel C). Similarly, the percentage proportion of the components of the average lifetimes for $\mathrm{C} 1$ and $\mathrm{C} 7$ changes at exactly the same pH values (see Fig. 4, Panels B and D). This clearly indicates substantially stronger aggregation of the C7 molecules, compared with $\mathrm{C} 1$, which has already been observed in the absorption spectra (Fig. 1, Panels A and C), where a significant reduction in the absorbance level was noted. This proves considerably better solubility of $\mathrm{C} 1$ in the aqueous medium with $\mathrm{pH}$ relevant from the physiological point of view.

The lengthening of the fluorescence lifetime in the case of molecules exhibiting the dual fluorescence effect is characteristic for charge-transfer systems and excimers [52], in contrast to processes induced by the phenomenon of aggregation (dimerisation), in which a clear reduction in the lifetime [27] or decay is observed.

\section{Resonance Light Scattering Study}

In order to relate the fluorescence effects observed with the molecular aggregation effects, respective Resonance Light Scattering (RLS, $\Delta \lambda=0$ ) spectra were obtained for $\mathrm{C} 1$ and 
C7 in the aqueous solution, depending on the changes in the medium $\mathrm{pH}$. Panels A and B in Fig. 5 demonstrate RLS spectra for C1 (Panel A) and C7 (Panel B) obtained at the different $\mathrm{pH}$ values of the medium. As reported in the literature (mainly by Pasternack and Parkash [48, 49]), the appearance of RLS bands should primarily be associated with chromophore aggregation of the systems present in the solution. Analysis of all the RLS spectra shown in Fig. 5 reveals the presence of RLS spectra (with higher or lower intensity) in the $\mathrm{pH}$ ranges where the dual fluorescence effect is observed for $\mathrm{C} 1$ and $\mathrm{C} 7$, as presented above in Fig. 1 (Panels B and D). Additionally, it can be noted that the increase in the $\mathrm{pH}$ value is accompanied by reduction of the RLS signal for both analysed analogues. Panel $\mathrm{C}$ in Fig. 5 shows the relationship between the RLS signal intensity and the solution $\mathrm{pH}$ for $\mathrm{C} 1$ (black line) and C7 (grey line). As can be noted, the RLS signal intensity substantially declines with the increase in $\mathrm{pH}$, which clearly suggests an impact of molecular aggregation on the spectral effects. The intensity of the signals is clearly higher for C7, where a substantial decline in the absorbance level was revealed by the measurements of absorption spectra. This evidences the impact of the alkyl substituent structure on the strength of the aggregation interactions in C7. The RLS signal loses its intensity at a $\mathrm{pH}$ value of ca. 7 for $\mathrm{C} 7$ and $\mathrm{C} 1$. The presence of the RLS spectra and their dependence on the changes in the $\mathrm{pH}$ of the analysed solutions clearly proves a relationship between the presented effect and the molecular aggregation of the investigated 1,3,4-thiadiazoles. It is also worth mentioning that the oscillatory structure of the RLS bands evidences the presence of various possibly differentsize aggregation structures of both $\mathrm{C} 1$ and $\mathrm{C} 7$, which also confirms the impact of the structure of the analysed analogues, in particular their alkyl substituents, on the observed effect (Scheme 1).

The observations clearly indicate a greater impact of molecular aggregation effects than the aforementioned TICT, ESIPT, and anti-Kasha processes or excimer fluorescence. Additionally, aggregation has a significant impact on changes in the electron charge distribution around the analysed molecules, which yields fluorescence spectral effects in a $\mathrm{pH}$ range. The difference in the structure of substituent groups of the analysed analogues (C1, C7) evokes different aggregation effects. Therefore, we postulate that the dual fluorescence effects in the 1,3,4-thiadiazoles are induced by at least two

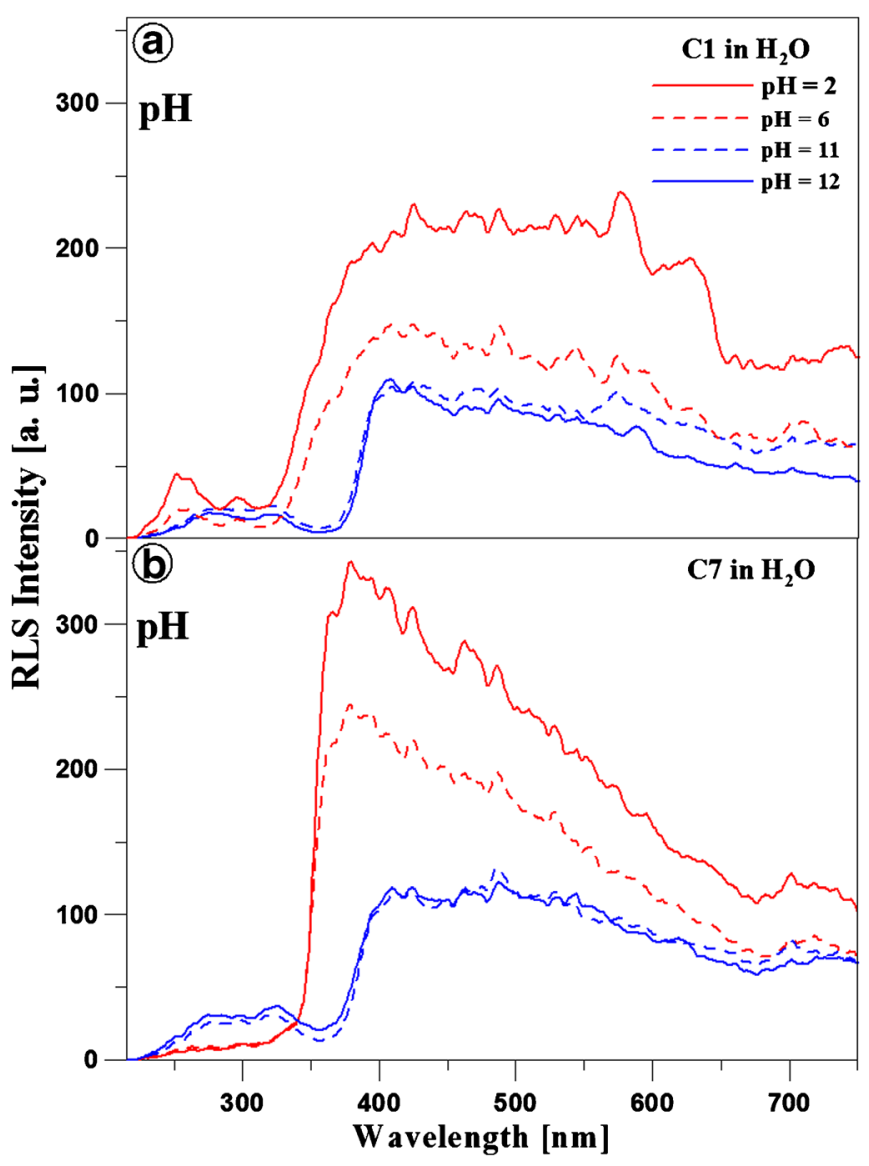

Fig. 5 Panels A and B: RLS spectra (Resonance Light Scattering, $\Delta \lambda=0$ ) for $\mathrm{C} 1$ and $\mathrm{C} 7$ obtained in the aqueous solution at the different $\mathrm{pH}$ values, presented in the figure for $\mathrm{pH} 1,3,7$, and 10 , respectively.

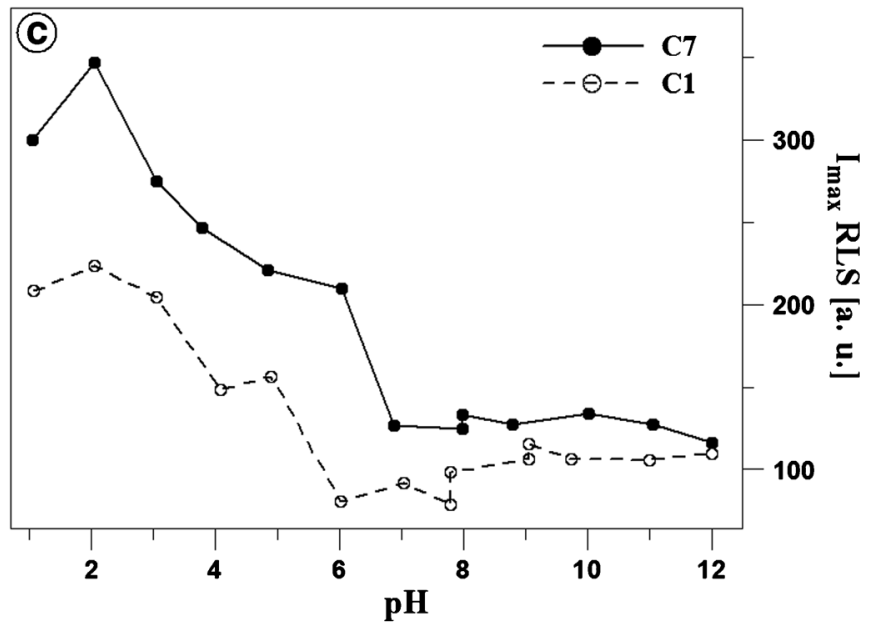

Panel C shows the ratio of the intensity of the RLS spectra for C1 (black line) and $\mathrm{C} 7$ (dashed grey line) depending on the $\mathrm{pH}$ of the aqueous solution. All RLS spectra were obtained at $T=23^{\circ} \mathrm{C}$ 
overlapping effects, i.e. molecular aggregation (depending on the substituent type in the molecule) and charge transfer CT (induced by the aggregation factor). Based on the spectral shifts in the electron absorption spectra, formation of card pack rather than head to tail aggregates can be assumed. Furthermore, the aggregation can be stronger at low, neutral, or very high $\mathrm{pH}$ values, whereas additional intermediate forms, which are a resultant of both ionised forms, can appear at neutral $\mathrm{pH}$. This can explain the rapid decline in the intensity of the RLS signal at neutral and high $\mathrm{pH}$ values. These considerations will be continued in further investigations of this highly attractive dual fluorescence spectroscopic effect in the 1,3,4-thiadiazole group with a resorcyl ring in the structure.

\section{Conclusions}

The results presented in this paper indicate appearance of the dual fluorescence effect in the fluorescence emission spectrum of the analysed 1,3,4-thiadiazole analogues $\mathrm{C} 1$ and $\mathrm{C} 7$ in the aqueous medium. In both compounds, this effect is most evident at the physiological $\mathrm{pH}$ and values lower that ca. 7 . Furthermore, the comparison of the analysed analogues with the previously described FABT compound, differing structurally by the presence of the amino $-\mathrm{N}-\mathrm{H}$ group in the substituent group and the fluorobenzene ring, revealed that there was no longwave fluorescence band with a maximum at ca. $500 \mathrm{~nm}$. In the case considered in this paper, a shortwave fluorescence emission band with a maximum at ca. $380 \mathrm{~nm}$ was found to accompany the decline in the $\mathrm{pH}$ value. This effect may be related to the structural differences (absence of the $\mathrm{N}-\mathrm{H}$ group in the $\mathrm{C} 1$ and $\mathrm{C} 7$ structure) between the compounds. In another study of a compound that is structurally similar to FABT but does not comprise the fluorobenzene fragment and differs from $\mathrm{Cl}$ only in the presence of the $\mathrm{N}$ $\mathrm{H}$ amine group, a band with a maximum at ca. $380 \mathrm{~nm}$ was observed at high $\mathrm{pH}$ values (results submitted for publishing). Acidification of the medium yielded a separate band at ca. $440 \mathrm{~nm}$ (as in the case of $\mathrm{C} 1$ and $\mathrm{C} 7$ at the high $\mathrm{pH}$ values). Therefore, there is an evident impact of the substituents in the 1,3,4-thiadiazole structure on the position of fluorescence bands despite the similar/identical chromophore organisation (five conjugated double bonds) in the analysed molecules. The lengthened fluorescence lifetime and change in the intensity of the RLS spectrum in the specified $\mathrm{pH}$ range for both molecules indicate association of this effect with the aggregation phenomenon dependent on the alkyl chain length. Therefore, it has been proposed that probably a combination of two effects, i.e. molecular aggregation (with the form dependent on the analogue) and CT charge transfer, is responsible for the observed fluorescence phenomena. This hypothesis is largely confirmed by the quantum-mechanical calculations
(TDDFT) performed for other analogues from this group of compounds. The non-specific interactions (e.g. formation of intra- and intermolecular hydrogen bonds or CT states) in the analysed 1,3,4-thiadiazole analogues modify the electronic structure surrounding the molecule, leading to changes in the distribution of electron density and thereby forcing CT charge transfer in the analysed analogues. This effect is reversible after alkalinisation of the medium and at high $\mathrm{pH}$ values (single fluorescence) and after acidification of the medium at $\mathrm{pH}$ in the range from 1 to ca. 7 (dual fluorescence).

The dual fluorescence effect occurs in a different energetic range than that in the 1,3,4-thiadiazoles analysed previously. In the theoretical aspect, it can be used for analysis of excitation states in these and other structurally similar molecules and for designing new fluorescence probes.

In conclusion, it should be emphasised that the analysed 1,3,4-thiadiazoles can serve as excellent fluorescent probes that are highly sensitive to changes in the medium $\mathrm{pH}$. Additionally, the attractiveness of the investigated systems is enhanced by their advantages in medical and pharmacological applications, which are more often based on new compounds with desirable properties.

Acknowledgements The research was partly supported by the grant from the University of Life Science in Lublin (TKF/MN/5 to AM).

Open Access This article is distributed under the terms of the Creative Commons Attribution 4.0 International License (http:// creativecommons.org/licenses/by/4.0/), which permits unrestricted use, distribution, and reproduction in any medium, provided you give appropriate credit to the original author(s) and the source, provide a link to the Creative Commons license, and indicate if changes were made.

\section{References}

1. Ma X, Yu H (2006) Global burden of cancer. Yale J Biol Med 79:85-94

2. Coleman CN, Minsky BD (2015) The verdict is in: the time for effective solutions to the global cancer burden is now. Lancet Oncol 16:1146-1147

3. Luszczki JJ, Karpińska M, Matysiak J, Niewiadomy A (2015) Characterization and preliminary anticonvulsant assessment of some 1,3,4-thiadiazole derivatives. Pharmacol Rep 67:588-592

4. Matysiak J, Nasulewicz A, Pelczynska M, Switalska M, Jaroszewicz I, Opolski A (2006) Synthesis and antiproliferative activity of some 5-substituted 2-(2,4-dihydroxyphenyl)-1,3,4thiadiazoles. Eur J Med Chem 41:475-482

5. Jäntschi L, Bolboacă S (2006) Modelling the inhibitory activity on carbonic anhydrase IV of substituted thiadiazole-and thiadiazolinedisulfonamides: integration of structure information. Electron $\mathrm{J}$ Biomed 2:22-33

6. Nadeem Siddiqui PA, Waquar Ahsan SN (2009) Pandeya, M Shamsher Alam thiadiazoles: progress report on biological activities. J Chem Pharm 1:19-30

7. Shawali AS (2014) 1,3,4-thiadiazoles of pharmacological interest: recent trends in their synthesis via tandem 1,3-dipolar cycloaddition: review. J Adv Res 5:1-17 
8. Rezki N, Al-Yahyawi AM, Bardaweel SK, Al-Blewi FF, Aouad MR (2015) Synthesis of novel 2,5-Disubstituted-1,3,4-thiadiazoles clubbed 1,2,4-Triazole, 1,3,4-thiadiazole, 1,3,4-oxadiazole and/or Schiff Base as potential antimicrobial and antiproliferative agents. Molecules 20:16048-16067

9. Farghaly TA, Abdallah MA, Masaret GS, Muhammad ZA (2015) New and efficient approach for synthesis of novel bioactive [1,3, 4]thiadiazoles incorporated with 1,3-thiazole moiety. Eur J Med Chem 97:320-333

10. Kovalenko SI, Nosulenko IS, Voskoboynik AY, Berest GG, Antypenko LN, Antypenko AN, Katsev AM (2012) Substituted $2-[(2-\mathrm{O} \times 0-2 \mathrm{H}-[1,2,4]$ triazino $[2,3-\mathrm{c}] q u i n a z o l i n-6-$ yl)thio]acetamides with thiazole and thiadiazole fragments: synthesis, physicochemical properties, cytotoxicity, and anticancer activity. Sci Pharm 80:837-865

11. Camoutsis C, Geronikaki A, Ciric A, Soković M, Zoumpoulakis P (2010) M., Z. Sulfonamide-1,2,4-thiadiazole derivatives as antifungal and antibacterial agents: synthesis, biological evaluation, lipophilicity, and conformational studies. Chem Pharm Bull (Tokyo) 58:160-167

12. Santagati M, Modica M, Santagati A, Russo F, Caruso A, Cutuli V, Di Pietro E, Amico-Roxas M (1994) Synthesis and pharmacological properties of benzothiazole, 1,3-4-oxadiazole and 1,3,4thiadiazole derivatives. Pharmazie 49:880-884

13. Gupta A, Mishra P, Pandeya SN, Kashaw SK, Kashaw V, Stables JP (2009) Synthesis and anticonvulsant activity of some substituted 1, 2,4-thiadiazoles. Eur J Med Chem 44:1100-1105

14. Tonew M (1976) Antiviral 1,3,4-thiadiazoles. II. Effects on cellular and viral RNA synthesis in Mengo-virus-infected FL cells. Chemotherapy 22:114-120

15. Alwan WS, Karpoormath R, Palkar MB, Patel HM, Rane RA, Shaikh MS, Kajee A, Mlisana KP (2015) Novel imidazo[2,1-b]-1, 3,4-thiadiazoles as promising antifungal agents against clinical isolate of Cryptococcus neoformans. Eur J Med Chem 95:514-525

16. Turner S, Myers M, Gadie B, Nelson AJ, Pape R, Saville JF, Doxey JC, Berridge TL (1988) Antihypertensive thiadiazoles. 1. Synthesis of some 2-aryl-5-hydrazino-1,3,4-thiadiazoles with vasodilator activity. J Med Chem 31:902-906

17. Haider S, Alam MS, Hamid H (2015) 1,3,4-thiadiazoles: a potent multi targeted pharmacological scaffold. Eur J Med Chem 92:156-177

18. Matwijczuk A, Gorecki A, Kaminski D, Mysliwa-Kurdziel B, Fiedor L, Niewiadomy A, Karwasz GP, Gagos M (2015) Influence of solvent polarizability on the keto-enol equilibrium in 4-[5-(naphthalen-1-ylmethyl)-1,3,4-thiadiazol-2-yl]benzene-1,3diol. J Fluoresc 25:1867-1874

19. Gagos M, Matwijczuk A, Kaminski D, Niewiadomy A, Kowalski R, Karwasz GP (2011) Spectroscopic studies of intramolecular proton transfer in 2-(4-fluorophenylamino)-5-(2,4dihydroxybenzeno)-1,3,4-thiadiazole. J Fluoresc 21:1-10

20. Matwijczuk A, Karcz D, Walkowiak R, Niewiadomy A, Wybraniec S, Karwasz G, Gagos M (2016) Keto-enol tautomerism of 2-(4fluorophenyl)-5-(2, 4-dihydroxyphenyl)-1, 3, 4-thiadiazole. Spectroscopic studies. Przem Chem 95:1894-1898

21. Matwijczuk A, Karcz D, Walkowiak R, Furso J, Gładyszewska B, Wybraniec S, Niewiadomy A, Karwasz GP, Gagoś M (2017) Effect of solvent polarizability on the keto/enol equilibrium of selected bioactive molecules from the 1, 3, 4-thiadiazole group with a 2, 4-hydroxyphenyl function. J Phys Chem A 121(7): $1402-1411$

22. Hoser AA, Kamiński DM, Matwijczuk A, Niewiadomy A, Gagoś M, Woźniak K (2013) On polymorphism of 2-(4fluorophenylamino)-5-(2,4-dihydroxybenzeno)-1,3,4-thiadiazole (FABT) DMSO solvates. Cryst Eng Comm 15:978-1988

23. Kamiński DM, Hoser AA, Gagoś M, Matwijczuk A, Arczewska M, Niewiadomy A, Woźniak K (2010) Solvatomorphism of 2-(4-
Fluorophenylamino)-5-(2,4-dihydroxybenzeno)-1,3,4-thiadiazole Chloride. Cryst Growth Des 10:3480-3488

24. Kaminski DM, Matwijczuk A, Pociecha D, Gorecka E, Niewiadomy A, Dmowska M, Gagos M (2012) Effect of 2-(4fluorophenylamino)-5-(2,4-dihydroxyphenyl)-1,3,4-thiadiazole on the molecular organisation and structural properties of the DPPC lipid multibilayers. Biochim Biophys Acta 1818:2850-2859

25. Kluczyk D, Matwijczuk A, Gorecki A, Karpinska MM, Szymanek M, Niewiadomy A, Gagos M (2016) Molecular Organization of Dipalmitoylphosphatidylcholine Bilayers Containing Bioactive Compounds 4-(5-heptyl-1,3,4-thiadiazol-2-yl) benzene-1,3-diol and 4-(5-methyl-1,3,4-thiadiazol-2-yl) benzene-1,3-diols. J Phys Chem B 120:12047-12063

26. Matwijczuk A, Kluczyk D, Górecki A, Niewiadomy A, Gagos M (2016) Solvent effects on molecular aggregation in 4-(5heptyl-1,3,4-thiadiazol-2-yl)benzene-1,3-diol and 4-(5-methyl1,3,4-thiadiazol-2-yl)benzene-1,3-diol. J Phys Chem B 120(32):7958-7969

27. Matwijczuk A, Kaminski D, Gorecki A, Ludwiczuk A, Niewiadomy A, Mackowski S, Gagos M (2015) Spectroscopic studies of dual fluorescence in 2-((4-fluorophenyl)amino)-5-(2, 4-dihydroxybenzeno)-1,3,4-thiadiazole. J Phys Chem A 119: 10791-10805

28. Karcz D, Matwijczuk A, Boroń B, Creaven B, Fiedor L, Niewiadomy A, Gagoś M (2017) Isolation and spectroscopic characterization of $\mathrm{Zn}(\mathrm{II}), \mathrm{Cu}(\mathrm{II})$, and $\mathrm{Pd}(\mathrm{II})$ complexes of 1,3,4thiadiazole-derived ligand. J Mol Struct 1128:44-50

29. Zhou P, Hoffmann MR, Han K, He G (2015) New insights into the dual fluorescence of methyl salicylate: effects of intermolecular hydrogen bonding and solvation. J Phys Chem B 119: 2125-2131

30. Yang W, Chen X (2014) Dual fluorescence of excited state intramolecular proton transfer of HBFO: mechanistic understanding, substituent and solvent effects. Phys Chem Chem Phys 16: 4242-4250

31. Prabhu AA, Sankaranarayanan RK, Venkatesh G, Rajendiran N (2012) Dual fluorescence of fast blue RR and fast violet B: effects of solvents and cyclodextrin complexation. J Phys Chem B 116: 9061-9074

32. Rajendiran N, Balasubramanian T (2007) Dual fluorescence of syringaldazine. Spectrochim Acta A Mol Biomol Spectrosc 68: 894-904

33. Suzuki N, Fukazawa A, Nagura K, Saito S, Kitoh-Nishioka H, Yokogawa D, Irle S, Yamaguchi S (2014) A strap strategy for construction of an excited-state intramolecular proton transfer (ESIPT) system with dual fluorescence. Angew Chem Int Ed Engl 53:8231-8235

34. Il'ichev YV, Kühnle W, Zachariasse KA (1998) Intramolecular charge transfer in dual fluorescent 4-(Dialkylamino)benzonitriles. Reaction Efficiency Enhancement by Increasing the Size of the Amino and Benzonitrile Subunits by Alkyl Substituents. J Phys Chem A 102:5670-5680

35. Monti F, Venturini A, Nenov A, Tancini F, Finke AD, Diederich F, Armaroli N (2015) Anilino-substituted Multicyanobuta-1,3-diene electron acceptors: TICT molecules with accessible conical intersections. J Phys Chem A 119:10677-10683

36. Deshmukh MS, Sekar N (2014) Novel twisted intramolecular charge transfer (TICT) extended fluorescent styryl derivatives containing quinoline electron releasing moiety. J Fluoresc 24: $1811-1825$

37. Padalkar VS, Seki S (2016) Excited-state intramolecular protontransfer (ESIPT)-inspired solid state emitters. Chem Soc Rev 45: 169-202

38. Manna A, Sayed M, Kumar A, Pal H (2014) Atypical energetic and kinetic course of excited-state intramolecular proton transfer 
(ESIPT) in room-temperature protic ionic liquids. J Phys Chem B 118:2487-2498

39. Li H, Niu L, Xu X, Zhang S, Gao F (2011) A comprehensive therotical investigation of intramolecular proton transfer in the excited states for some newly-designed diphenylethylene derivatives bearing 2-(2-Hydroxy-phenyl)-Benzotriazole part. J Fluoresc 21: $1721-1728$

40. Gao F, Ye X, Li H, Zhong X, Wang Q (2012) Evidence for twophoton absorption-induced ESIPT of chromophores containing hydroxyl and Imino groups. ChemPhysChem 13:1313-1324

41. Ding G, Lu Y, Gong Y, Ma L, Luo Z, Zhang S, Gao F, Li H (2016) New AB2 type two-photon absorption dyes for well-separated dualemission: molecular preorganization based approach to photophysical properties. Tetrahedron 72:3040-3056

42. Ding G, Lu Y, Qin X, Su J, Zhang S, Li H, Luo Z, Chen L, Gao F (2017) New organic conjugated dye nano-aggregates exhibiting naked-eye fluorescence color switching. Dyes Pigments 139:19-32

43. Ito F, Kakiuchi T, Sakano T, Nagamura T (2010) Fluorescence properties of pyrene derivative aggregates formed in polymer matrix depending on concentration. Phys Chem Chem Phys 12: 10923-10927

44. Haedler AT, Misslitz H, Buehlmeyer C, Albuquerque RQ, Köhler A, Schmidt a HW (2013) Controlling the n-Stacking Behavior of Pyrene Derivatives:Influence of H-Bonding and Steric Effects in DifferentStates of Aggregation. Chem Phys Chem 14:1818-1829

45. Waluk J (2006) Ground- and excited-state tautomerism in porphycenes. Acc Chem Res 39:945-952
46. Ziolek M, Kubicki J, Maciejewski A, Naskrecki R, Grabowska A (2006) Enol-keto tautomerism of aromatic photochromic Schiff base N,N'-bis(salicylidene)-p-phenylenediamine: ground state equilibrium and excited state deactivation studied by solvatochromic measurements on ultrafast time scale. J Chem Phys 124:124518

47. Brancato G, Signore G, Neyroz P, Polli D, Cerullo G, Abbandonato G, Nucara L, Barone V, Beltram F, Bizzarri R (2015) Dual fluorescence through Kasha's rule breaking: an unconventional Photomechanism for intracellular probe design. J Phys Chem B 119:6144-6154

48. Parkash J, Robblee JH, Agnew J, Gibbs E, Collings P, Pasternack RF, de Paula JC (1998) Depolarized resonance light scattering by porphyrin and chlorophyll a aggregates. Biophys J 74:2089-2099

49. Pasternack R, Collings P (1995) Resonance light scattering: a new technique for studying chromophore aggregation. Science 269: 935-939

50. Kasha M (1963) Energy transfer mechanisms and the molecular exciton model for molecular aggregates. Radiat Res 20:55-71

51. Kasha M, Rawls HR, Ashraf El-Bayoumi M (1965) The exciton model in molecular spectroscopy. Pure Appl Chem 11:371-392

52. Bains GK, Kim SH, Sorin EJ, Narayanaswami V (2012) Extent of pyrene excimer fluorescence emission is a reflector of distance and flexibility: analysis of the segment linking the LDL receptorbinding and Tetramerization domains of apolipoprotein E3(). Biochemistry 51:6207-6219 